$\begin{array}{cc}\text { ACADEMIA ROMÂNĂ } & \text { Rev. Roum. Chim., } \\ \mathbf{2 0 2 1 , 6 6 ( 1 ) , 6 5 - 7 4}\end{array}$

\title{
PEAK COMPRESSION INDUCED BY LARGE VOLUME INJECTION OF HIDROPHOBIC ALCOHOLS IN REVERSED-PHASE LIQUID CHROMATOGRAPHY
}

\author{
Elena BACALUM, ${ }^{\mathrm{a}}$ Toma GALAON,${ }^{\mathrm{b}}$ Victor DAVID ${ }^{\mathrm{a},{ }^{*}}$ and Serban C. MOLDOVEANU ${ }^{\mathrm{c}}$ \\ ${ }^{a}$ University of Bucharest, Faculty of Chemistry, Department of Analytical Chemistry, Sos. Panduri, no 90, Bucharest 050663, \\ Roumania \\ ${ }^{\mathrm{b}}$ National Research and Development Institute for Industrial Ecology-ECOIND, Drumul Podul Dambovitei Street, 71-73, Sector 6, \\ Bucharest, Roumania \\ c J. Reynolds Tobacco Co., 950 Reynolds Blvd. Winston-Salem, NC 27105, USA
}

Received December 3, 2020

Injections of high volumes of samples containing hydrophobic diluents are possible in reversed-phase liquid chromatography, and in some cases an effect of peak compression can be observed. Three classes of organic compounds were studied as target compounds for peak compression: pharmaceuticals (pentoxifylline; salicylic acid; caffeine, ethylparaben); pollutants (simazine, atrazine), and natural compounds from tabacoo provenience (nicotine). Five aliphatic alcohols from butanol to octanol as hydrophobic solvents were used as sample diluent in the view of influencing the peak efficiency for the studied analytes. Among them, only pentanol, hexanol and heptanol were observed to produce peak compression resulting in a very high chromatographic efficiency for the studied compounds,

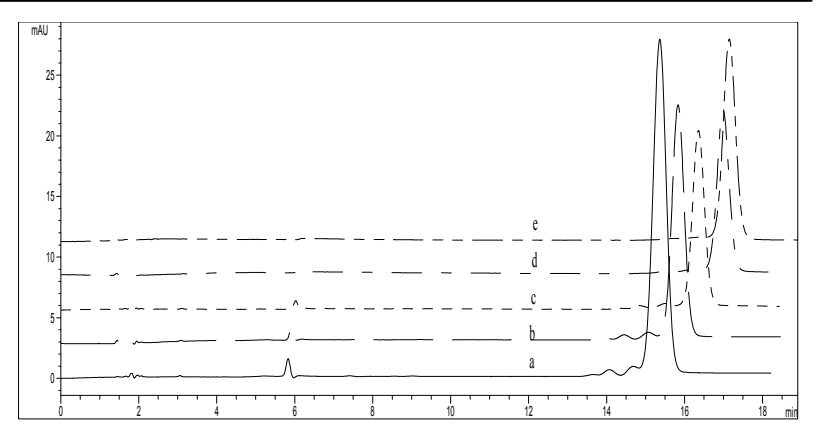
while butanol and octanol allowed large volume injection with gradual decrease of the retention time of the dissolved analytes, but without their peak compression. Pentoxifylline, salicilic acid, simasine, are some examples of analytes that are characterized by sharp chromatographic peaks (high chromatographic efficiency, with over 10,000 plates/column), when high volumes of pentanol or hexanol $(50-100 \mu \mathrm{L})$ solutions are injected. Chromatogram monitoring was performed by UV-spectrometry detection or refractive index detection.

\section{INTRODUCTION}

Sample preparation is considered the most manpower consuming and significant source of errors in a chromatographic analysis. ${ }^{1-3}$ Liquid-liquid extraction (LLE) techniques are comonly utilized approaches for sample preparation, in environmental ${ }^{4-6}$ and pharmaceutical analysis, ${ }^{7-9}$ or in bioanalysis. ${ }^{10-12}$ In most cases the organic layer generated by liquid-liquid extraction is prelevated and concentrated before the chromatographic analysis. A new possibility avoiding the solvent evaporation for sample concentraion is to inject directly high volumes of the organic solvent in order to load larger (detectable) amounts of extracted species. $^{13}$ Examples of non-miscible sovents usefull in LLE and applied in liquid chromatography by large volume injection are

\footnotetext{
${ }^{*}$ Corresponding author: victor.david@chimie.unibuc.ro
} 
aliphatic $c^{14-16}$ or aromatic ${ }^{17}$ hydrocarbons, aliphatic alcohols, ${ }^{18,19}$ chlorinated solvents, ${ }^{20}$ or "green" solvents. ${ }^{21}$ The principle of this approach applied to the reversed-phase liquid chromatography (RPLC) relies on the adsorption of the sample diluent (solvent) onto the surface of the stationary phase at the head of the chromatographic column, while the dissolved analytes participate to the retention process on the rest of the stationary phase. ${ }^{13,14}$ The sample solvent also elutes eventually from the column head and act as a "compressing" eluent for the analytes. Consequently, by high volume injection the analytes elute at smaller retention time than resulted from the injection of typical injection volumes (i.e., $1-10 \mu \mathrm{L}$ ). Several advantages of this approach in the chromatographic analysis include improved sensitivity, less analysis time and a higher precision by eliminating the error source caused by the solvent evaporation for sample concentration. ${ }^{15,18}$

However, the elution process when injecting large sample volumes is more complex, and some circumstances revealed that the shape of the chromatographic peaks can be disturbed. In other cases, the peaks are sharp, and consequently more desirable for the chromatographic analysis. ${ }^{19}$ This last situation can be considered as peak compression. Peak compression may occur also under linear gradient elution and this possibility has been already reported ${ }^{22,23}$ and explained in the literature. ${ }^{24,25}$ Narrow peaks are advantageous for peak integration for quantitative analysis and they improve the integration of chromatographic peaks at the detection limit. It is the aim of this study to investigate the effects of high volume injection of hydrophobic aliphatic alcohols as sample diluent on the peak shape and chromatographic efficiency, and to emphasize the role of the mobile phase and sample diluent on the effect of peak compression.

\section{EXPERIMENTAL}

\section{Materials and Reagents}

Butanol, 1-pentanol, 1-hexanol, 1-heptanol, octanol, and methanol of HPLC grade were from Sigma-Aldrich (Germany). Phosphoric acid (pro-analysis grade) was obtained from Merck (Germany). Water for chromatography was obtained within the laboratory with a TKA Lab HP 6UV/UF instrument. The studied compounds (pentoxifylline, salicilic acid; caffeine, ethylparaben, simazine) were purchaised from Merck (Germany). Nicotine was obtained from Toronto
Research Chemicals Inc. (TRC) (North York, ON, Canada).

Initial standard solutions of each studied compound were prepared in one of the studied hydrophobic solvents (butanol, pentanol, hexanol, heptanol and octanol) in a concentration of $200 \mathrm{ng} / \mathrm{mL}$. These solutions were further diluted, such that the amount of analyte loaded into the chromatographic column was kept constant at $200 \mathrm{ng}$ by modifying accordingly the analyte concentration.

\section{Instrumentation}

The chromatographic experiments were performed with an Agilent 1100 Series liquid chromatograph (Agilent Technologies) in the following configuration: degasser (G1379A), binary pump (G1312A), auto sampler (G1313A), column thermostat (G1316A), and diode array detector (G1315A). Chromatographic data were acquired by means of Agilent Chemstation software rev. B.01.03. For the analysis of nicotine, aditionally a refractive index detector (G1362A) was utilized.

New and tested chromatographic columns were used having the following provenience and characteristics: Ultisil XB C18 (Welch Technologies) with $150 \mathrm{~mm}$ length, $4.6 \mathrm{~mm}$ inner diamter, $5 \mu \mathrm{m}$ particle size, and Xterra RP18 (Waters) with $150 \mathrm{~mm}$ length, $4.6 \mathrm{~mm}$ inner diamter, $5 \mu \mathrm{m}$ particle size. In the experiments, the evaluation of nicotine peak compression was performed only on Xterra column, and most other studies were performed only on the Ultisil column. Caffeine and pentoxyfilline were studied on both columns.

\section{Experimental procedure}

Injection with set-up volumes were applied automatically and the chromatograms were aquired at wavelength specific to each studied compound. After the chromatographic run, a post-elution program was applied in order to clean the column with methanol (20 minutes) from the retained alcohol, and acomodate the column for the next run (15 minutes with mobile phase composition used in the next experiment). Each chromatographic run was carried out at a constant temperature of $25^{\circ} \mathrm{C}$.

Mobile phase compositions was based on methanol as organic modifier and water, and detailes are further described when the experimental results are discussed.

\section{Evaluation of column efficiency}

Column efficiency is caracterized by the theoretical plate number and depends on the column characteristics, but also on the compound used in the separation. The expression of the theoretical plate number $N$ is given by the formula:

$$
N=16 t_{R}^{2} / W_{b}^{2}
$$

where $t_{R}$ is the retention time of the evaluated compound and $W_{b}$ is the peak width at the peak base which is the distance (in time) between the points of intersection of baseline with the tangents to the Gaussian shape of the peak at the inflexion points, as shown in Fig. 1.

Column efficiency being compound dependent and characterized by the parameters of a specific peak, can also be indicated as peak efficiency. 


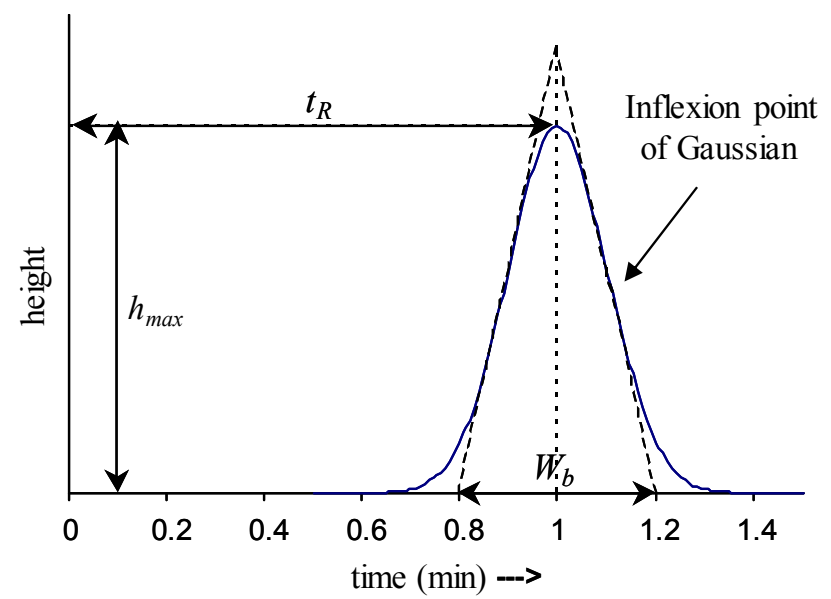

Fig. 1 - Measurement of the peak width $W_{b}$ at the base of chromatogram ${ }^{26}$.

\section{RESULTS AND DISCUSSION}

The possibility of injecting high volume of hydrophobic alcohols is achievable when the mobile phase has a large content in aqueous component. Otherwise, the sample diluent will become rapidly miscible with mobile phase and the elution process will be complicated by this phenomenon. Therefore, in this study in almost all cases mobile phase compositions consisted of a low concentration in water of the organic modifier (namely, methanol) to avoid miscibility. This condition has been already reported for other hydrophobic injection solvents, such as aliphatic, or aromatic hydrocarbons. ${ }^{13-18}$ On the other hand, for large content of water in the mobile phase composition, the retention of injected compounds on the stationary phase surface is acceptable. By using hydrophobic alcohols (butanol to octanol) as sample diluent, the methanol was preferred as organic modifier in the composition of the mobile phase. Experiments carried out with mobile phases containing acetonitrile in its composition did not produce similar chromatographic results as those obtained by using methanol.

According to the principle previously mentioned the retention time $\left(t_{\mathrm{R}}-\right.$ minutes $)$ of the dissolved analytes decreases by the increase of the injection volume $\left(V_{\mathrm{inj}}\right)$. The dependence between $t_{\mathrm{R}}$ and $V_{\text {inj }}$ can be described a polynomial equation $\left(t_{\mathrm{R}}=a+b V_{\mathrm{inj}}+c V_{\mathrm{inj}}^{2}\right)$, where $a, b$ and $c$ are regression parameters.

A series repeated experiments carried out for target analytes, such as those mentioned in the Experimental section, revealed in some cases an unusual effect, namely the chromatographic peaks having a very sharp shape. This can be conveniently described by peak efficiency. Injection of butanol or octanol showed that in almost all situations the value of $N$ was below 5,000 , and not considered as compressed peaks. Pentanol, hexanol and heptanol showed that the peaks corresponding to some analytes dissolved in these solvents are compressed, resulting in high peak efficiency $(N>5,000)$. These situations are further described and discussed.

A first example is given by the elution process of pentoxifylline injected in hydrophobic alcohols on a Ultisil XB C18 column. For a mobile phase composition of $25 \%$ methanol and $75 \%$ aqueous component $\left(0.1 \% \mathrm{H}_{3} \mathrm{PO}_{4}\right)$, a clear trend is noticed for the increase of the chromatographic efficiency of pentoxifylline dissolved in hexanol by increasing the injected sample volume in the column. At the same time, the retention time decreased with the increase of sample volume with a dependence depicted in Fig. 2.

A same behaviour as for pentoxifylline was noticed for mobile phase consisting in $30 \% \mathrm{MeOH}$ and $70 \%$ aquoues component (Fig. 3), when the peak efficiency reached the highest value, of more than 350,000 plates/column. By changing the sample diluent (hexanol) with heptanol the peak efficiency decreased linearly from 11,419 to 9561 by increasing the injected volume in the chromatographic column from 10 to $100 \mu \mathrm{L}$. In case of pentanol as sample diluent of pentoxifylline a random variation of efficiency was observed. A similar behavior has been observed for ethylparaben (Fig. 4), with peak compression resulted for the same sample solvent as for pentoxifylline. For ethylparaben, the efficiency was smaller, but it was over 10,000 plates/column, with a maximum of about 55,000 plates/column obtained for $50 \mu \mathrm{L}$ injection volume in hexanol. The retention of ethylparaben was described by the polynomial dependence between $t_{\mathrm{R}}$ and $V_{\mathrm{inj}}$ as following: $t_{R}=21.41-0.356 V_{i n j}+2.28 \times 10^{-3} V_{i n j}^{2}$. 

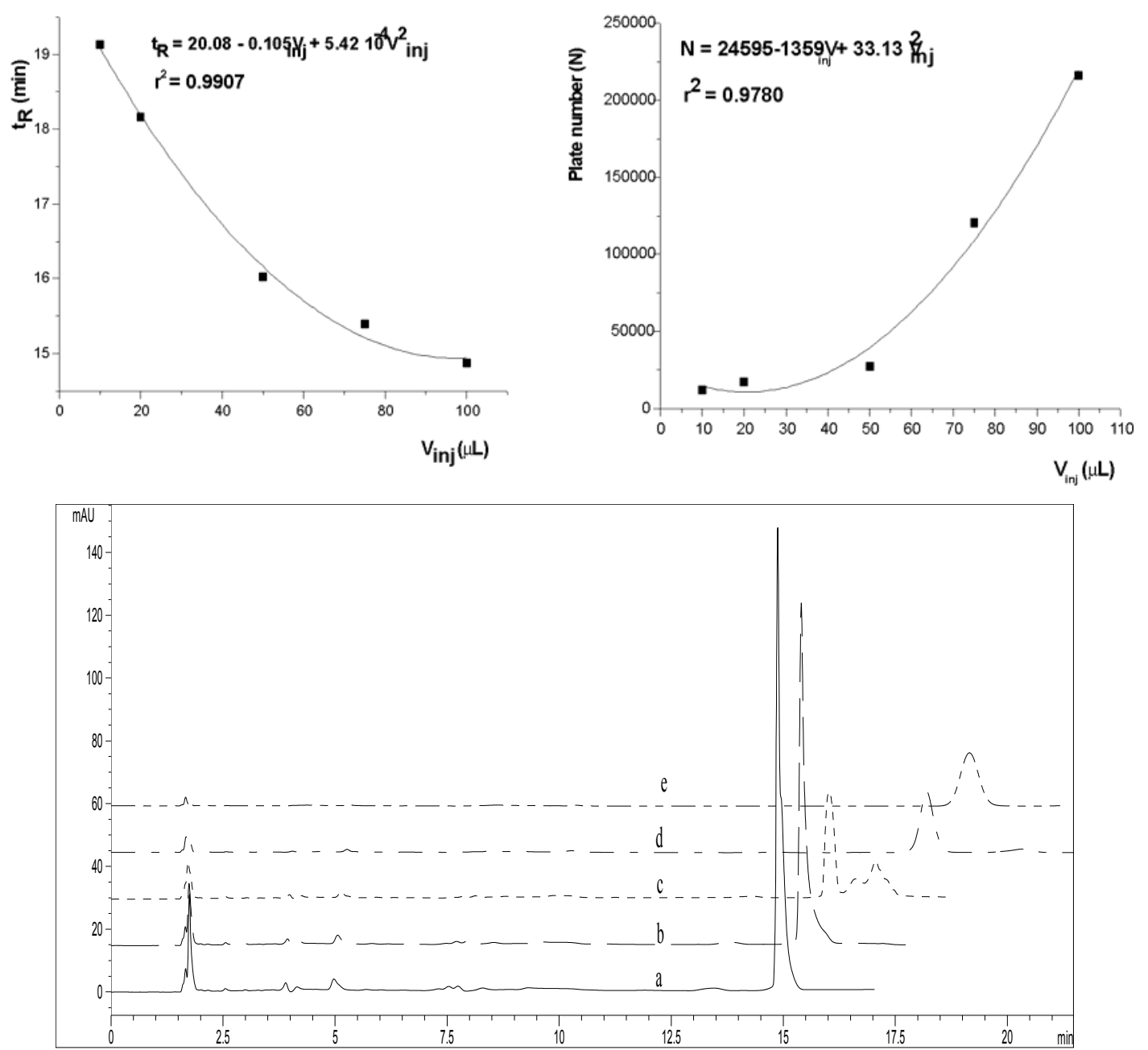

Fig. 2 - Overlapped chromatograms illustrating the plate number increase for pentoxifylline peak with the increase of injection volume from 10 to $100 \mu \mathrm{L}$ using 1-hexanol as diluent. Chromatographic conditions: mobile phase aq $0.1 \% \mathrm{H}_{3} \mathrm{PO}_{4} / \mathrm{MeOH}=70 / 30(\mathrm{v} / \mathrm{v})$. Injected volume of pentoxifylline in 1-hexanol is (a) 100; (b) 75; (c) 50 ; (d) 20 and (e) $10 \mu \mathrm{L}$.

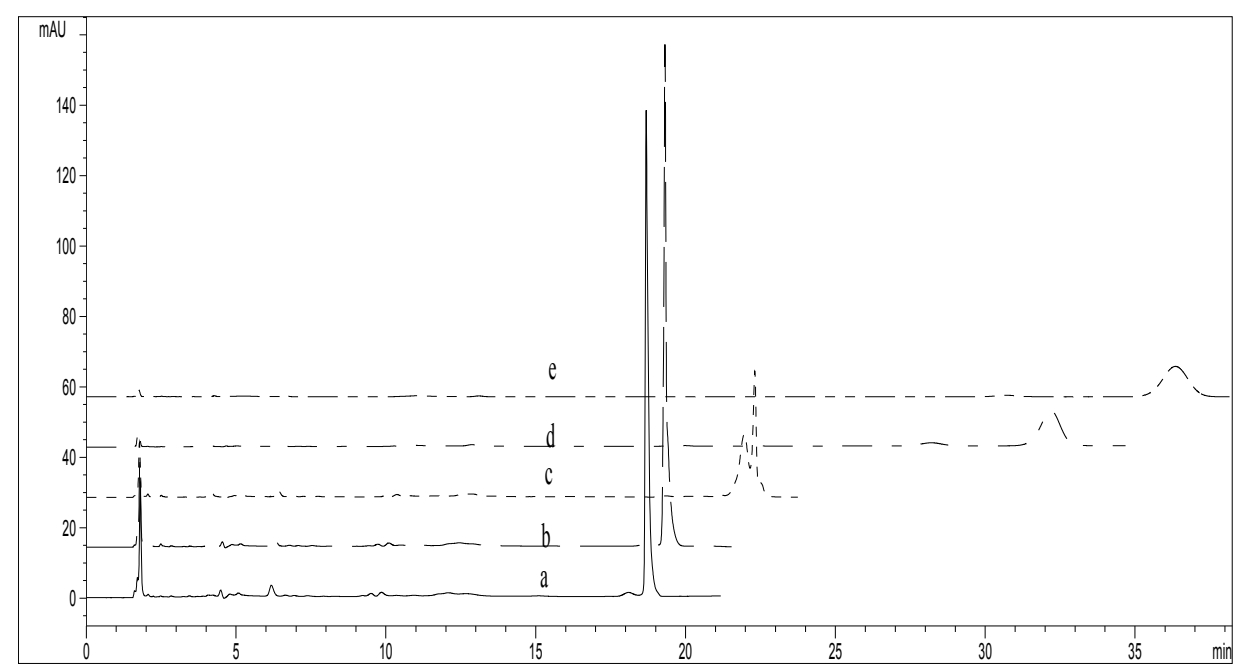

Fig. 3 - Overlapped chromatograms illustrating the plate number increase for pentoxifylline peak with the increase of injection volume from 10 to $100 \mu \mathrm{L}$ using 1-hexanol as diluent. Chromatographic conditions: mobile phase aq $0.1 \% \mathrm{H}_{3} \mathrm{PO}_{4} / \mathrm{MeOH}=75 / 25(\mathrm{v} / \mathrm{v})$. Injected volume of pentoxifylline in 1-hexanol is (a) 100 , (b) 75 , (c) 50 , (d) 20 and (e) $10 \mu \mathrm{L}$. Calculated plate number for pentoxifylline is (a) 174,895; (b) 389,763; (c) split peak; (d) 11,276; (e) 8,257. 


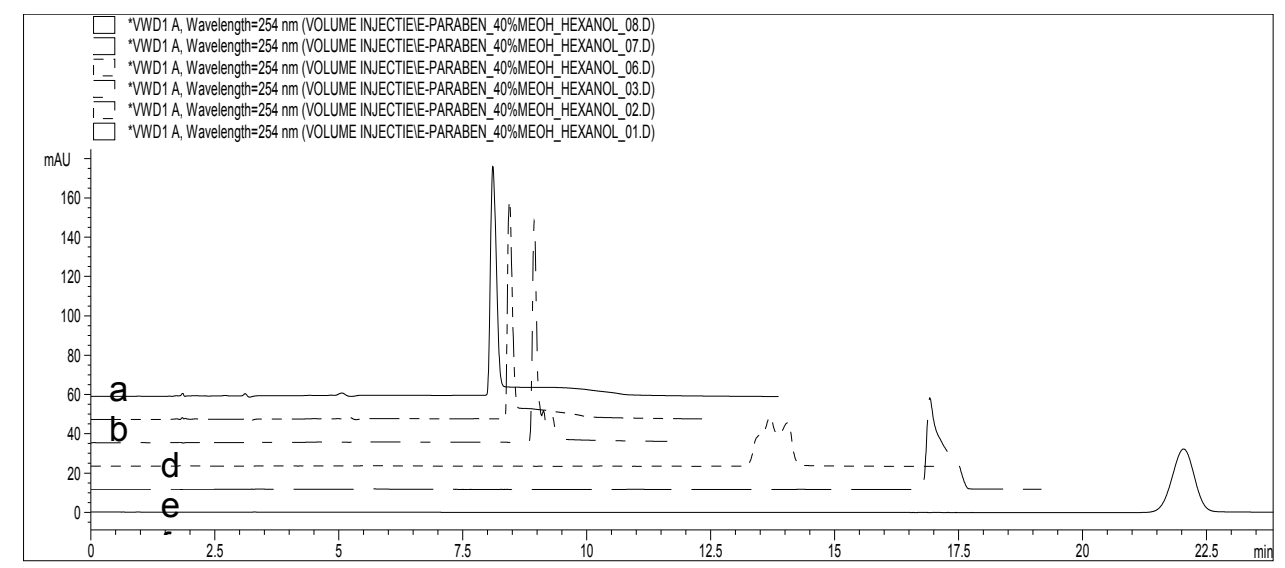

Fig. 4 - Overlapped chromatograms illustrating the plate number increase for ethylparaben peak with the increase of injection volume from 1 to $100 \mu \mathrm{L}$ using 1-hexanol as diluent. Chromatographic conditions: mobile phase aq $0.1 \% \mathrm{H}_{3} \mathrm{PO}_{4} / \mathrm{MeOH}=60 / 40(\mathrm{v} / \mathrm{v})$. Injected volume of ethylparaben in 1-hexanol is (a) 100 ; (b) 75 ; (c) 50 ; (d) 20 ; (e) $10 \mu \mathrm{L}$; (f) $1 \mu \mathrm{L}$. Calculated plate number for ethylparaben is (a) 20,190; (b) 35,430 (c) 54,940; (d) splitted peak; (e) 15,224; f) 11,421 plates/column.

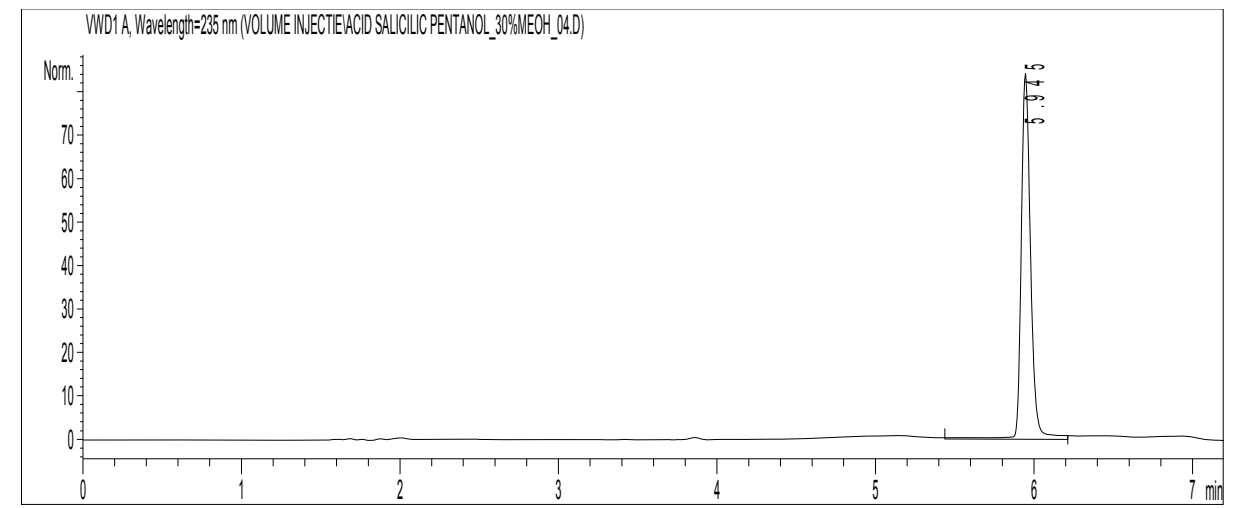

Fig. 5 - The chromatogram resulted after injection of $75 \mu \mathrm{L}$ sample containing salicylic acid in pentanol; mobile phase: $30 \% \mathrm{MeOH}$ and $70 \%$ aqueous compoent $\left(0.1 \% \mathrm{H}_{3} \mathrm{PO}_{4}\right)$; peak efficiency: $\mathrm{N}>54,000$ plates/column.

Another studied analyte that was involved in peak compression was salicylic acid, but compression occured only in pentanol as sample diluent. The dependence of efficiency did not follow a polynomial increase with the injected sample volume as observed for pentoxifylline, but this parameter was highest, with a maximum of $\mathrm{N}=54,657$ plates/column, for an injection volume of $75 \mu \mathrm{L}$ (Fig. 5). The other tested solvents were not involved in effects of peak compression, and in many cases the peak of this analyte was splitted or characterized by an efficiency $\mathrm{N}<1,000$ plates/column.

Efficiency of the caffeine peak decreased with the increase of the sample injection volume when haxanol was used as sample diluent, but was maintained close to 10,000 plates/column (Fig. 6). A similar trend was observed for caffeine when heptanol was used as sample diluent, but the efficency was significantly higher than for hexanol, with $\mathrm{N}$ varrying between 13,000 plates/column for an injection volume of $10 \mu \mathrm{L}$ and 10,400 plates/column for an injection volume of $100 \mu \mathrm{L}$. The same effect was observed for simazine when pentanol was used as sample diluent, but when switching from hexanol to heptanol as diluent of the simazine, it was observed a linear decrease of peak efficiency from 10450 to 6194 when increasing the injected volume in the chromatographic column from 5 to $100 \mu \mathrm{L}$.

Also, the peak of simazine showed a high efficiency, and some representative chromatograms obtained by injecting high volumes of samples in pentanol or hexanol as diluents are shown in Fig. 7 and Fig. 8. In both cases, the mobile phase composition was 30/70, v/v (methanol/aqueous with $0.1 \% \mathrm{H}_{3} \mathrm{PO}_{4}$ ). The peak efficiency was high for simazine in case of of using pentanol as sample diluent and only for high injection volumes $(75$ and $100 \mu \mathrm{L})$. In this case, 
peak efficiency as shown in Fig. 8 was highre than 100,000 , and 200,000 plates/column, respectively, for the two mentioned injection volumes. For lower injection volumes the chromatographic peak of this compound was distorsed or splitted, which show that peak compression takes place only for high injection volumes of sample. The same effect was observed for simazine when pentanol was replaced by hexanol, but no peak compression was observed for heptanol and octanol.

In case of ethylparaben, peak compression was observed only for haxanol as sample diluent injected for volumes of 50,75 and $100 \mu \mathrm{L}$, but the peak efficiencies were lower than in case of the other studied compounds. Their values decresead with the increase of the injection volume, in the order: 54,940 (for $50 \mu \mathrm{L}$ ); 35,430 (for $75 \mu \mathrm{L}$ ); 20,190 (for $100 \mu \mathrm{L}$ ). For average injection volumes of 10 and $20 \mu \mathrm{L}$ the peak of ethylparaben was splitted, as can be seen from Fig. 9, where overlapped chromatograms for different volumes of injected samples containing ethylparaben in hexanol are shown.

In case of nicotine, peak compression was observed for pentanol at $100 \mu \mathrm{L}$ injection with a concentration of $15 \mu \mathrm{g} / \mathrm{mL}$ and detection at 254 $\mathrm{mn}$, on a Xterra RP18 column. The separation was performed in gradient conditions with water/methanol from $4 \%$ to $50 \%$ methanol linear increase. The chromatogram is shown in Fig. 10.

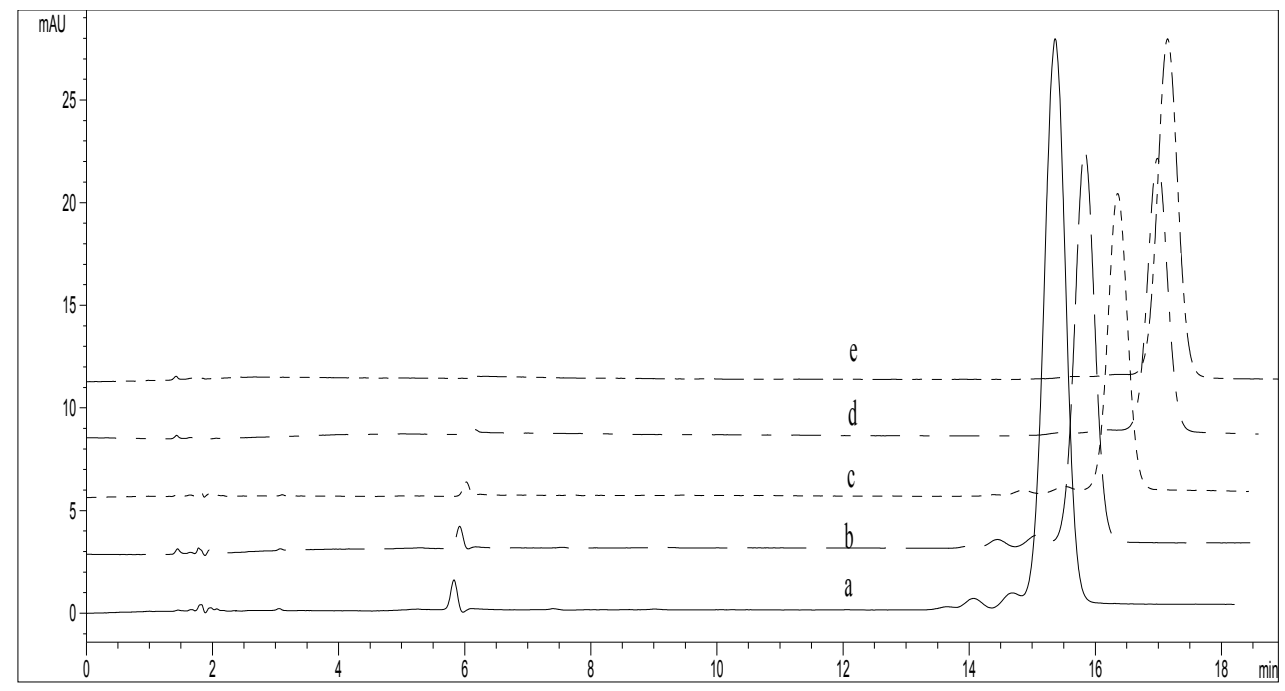

Fig. 6 - Overlapped chromatograms illustrating the plate number increase for caffeine peak with the increase of injection volume from 10 to $100 \mu \mathrm{L}$ using 1-hexanol as diluent. Chromatographic conditions: mobile phase aq $0.1 \% \mathrm{H}_{3} \mathrm{PO}_{4} / \mathrm{MeOH}=82 / 18(\mathrm{v} / \mathrm{v})$. Injected volume of caffeine in 1-hexanol is (a) 100 , (b) 75 , (c) 50 , (d) 10 , e) $5 \mu \mathrm{L}$. Calculated plate number for simazine is (a) 9,362 ; (b) 11,372 ; (c) 12,937 ; (d) 13,650 ; e) 13,337 .

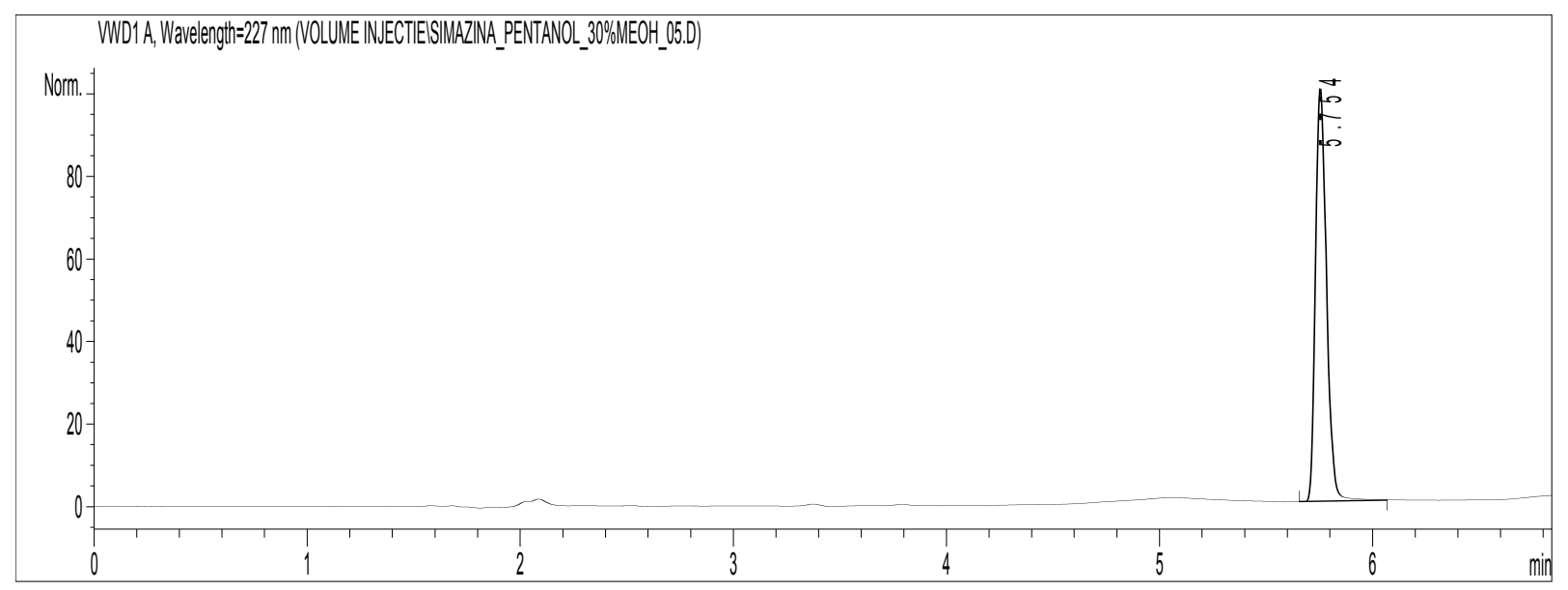

Fig. 7 - The chromatogram resulted after injection of $100 \mu \mathrm{L}$ sample containing simazine in pentanol; mobile phase: $30 \% \mathrm{MeOH}$ and $70 \%$ aqueous compoent $\left(0.1 \% \mathrm{H}_{3} \mathrm{PO}_{4}\right)$; peak efficiency: $\mathrm{N}>64,000$ plates/column. 


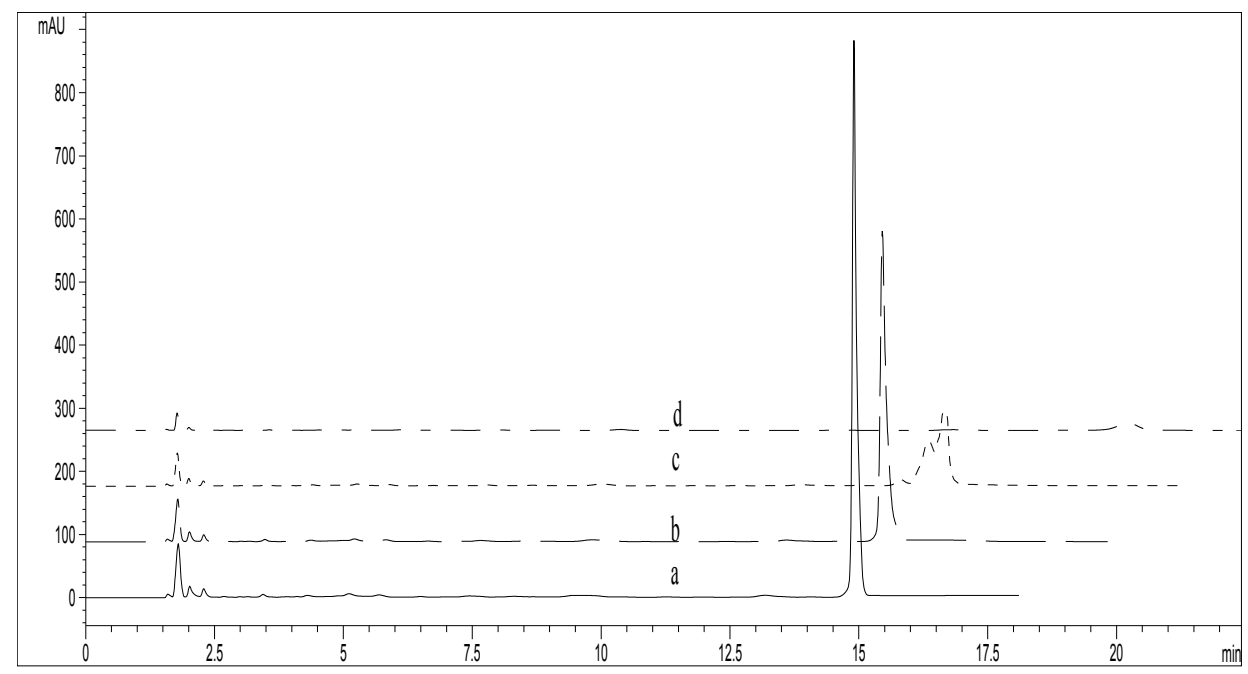

Fig. 8 - Two overlapped chromatograms illustrating the high efficiency increase for simazine peak for the injection volume of $100 \mu \mathrm{L}$ (a) and $75 \mu \mathrm{L}$ (b) using hexanol as diluent. Chromatographic conditions: mobile phase aq $0.1 \% \mathrm{H}_{3} \mathrm{PO}_{4} / \mathrm{MeOH}=70 / 30(\mathrm{v} / \mathrm{v})$. Calculated plate number for simazine is (a) 106,179 ; (b) 217,466 .

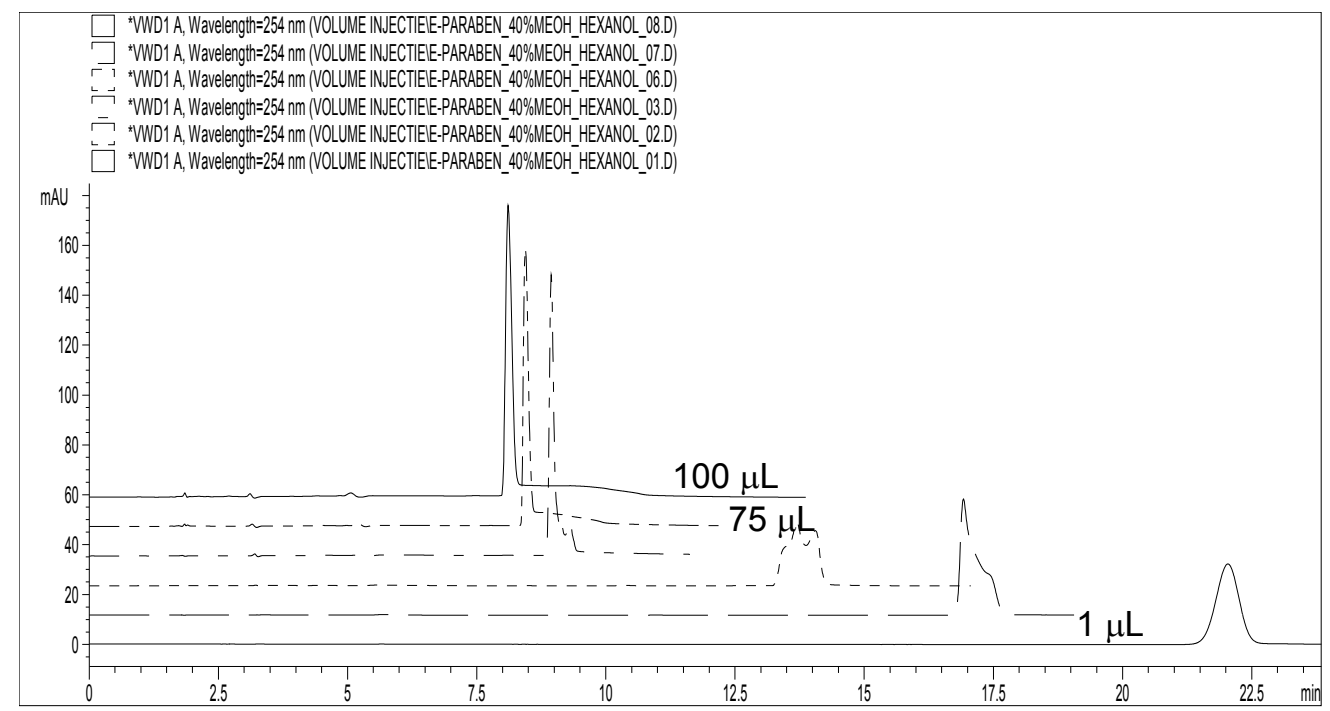

Fig. 9 - Six overlapped chromatograms illustrating the high efficiency increase for ethylparaben peak for the injection volume of $100 \mu \mathrm{L}$ (a) and $75 \mu \mathrm{L}$ (b) using hexanol as diluent and peak splitting (mobile phase containing $40 \%$ methanol and $60 \%$ aqueous solution of $0.1 \% \mathrm{H}_{3} \mathrm{PO}_{4}$.

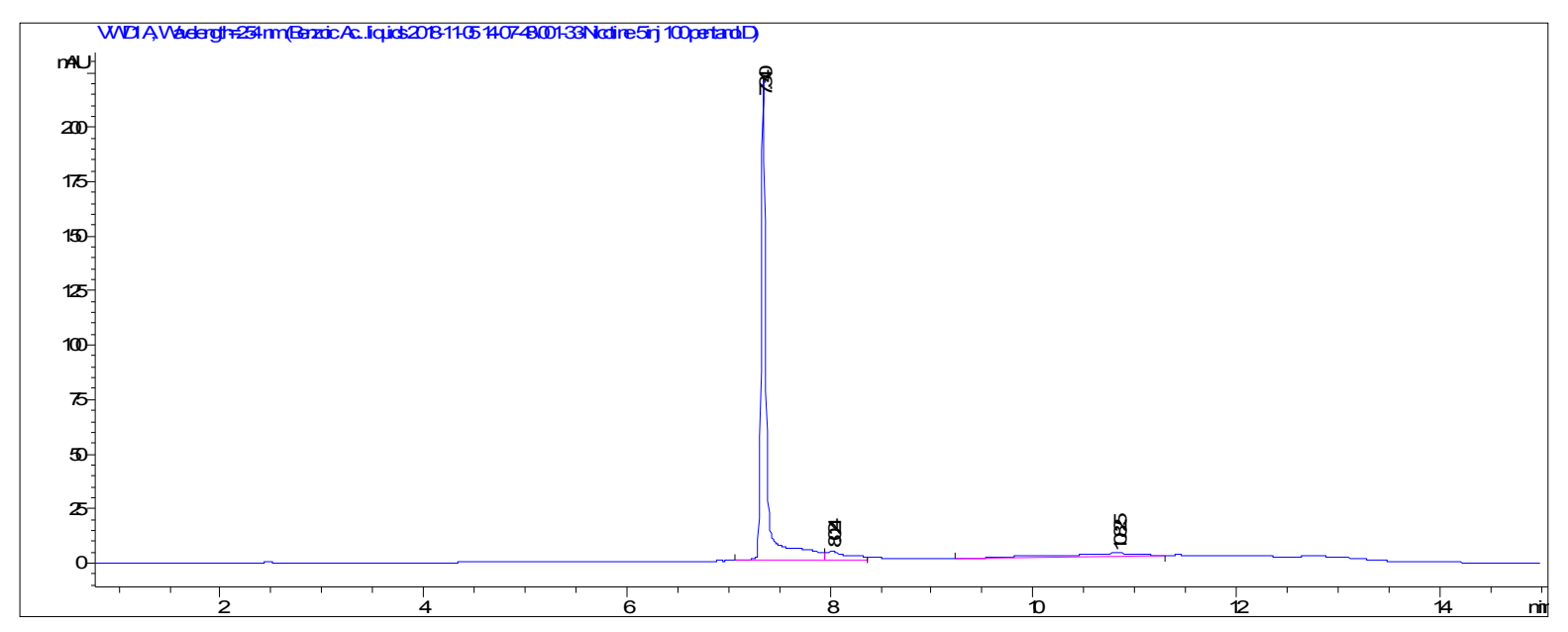

Fig 10 - Chromatogram of a nicotine peak obtained fro $100 \mu \mathrm{L}$ injection of a pentanol solution containing $15 \mu \mathrm{g} / \mathrm{mL}$ nicotine. 
In order to explain the different behaviours of analytes in different injection solvents and volumes some HPLC separations were performed using a second detector for observing the front of solvent eluting from the chromatographic column, which can not be detected by UV absorption. This procedure has already for the visualization of the octanol front eluted from column and its effect on the peak shape of a target solute (indapamide) for different mobile phase compozitions. ${ }^{18}$

For example, a high volume of pentanol $(100 \mu \mathrm{L})$ containing caffeine and pentoxyfilline was injected using water/methanol $65 / 35(\mathrm{v} / \mathrm{v})$ as mobile phase in isocratic conditions. The two chromatograms for both types of detection is shown in Fig. 11. Upper trace indicated the response of refractive index detector, which shows that pentanol is eluting from the chromatographic column after 4 minutes. The lower trace that of UV detector at $254 \mathrm{~nm}$ indicates that caffeine and pentoxyfilline are eluted at the front of the sample solvent peak. Similar results were obtained for the same analytes injected in hexanol as sample diluent, when pentoxyfilline eluted from the chromatographic column very close to the front of sample diluent eluting from the column. This could be the cause of the compression of this compound, while the peak of caffeine is not influenced by the diluent front. The diluent front eluting from the chromatographic column is likely to produce a uncontrolled gradient of mobile phase concentration composed of water, methanol and the sample diluent. This gradient may compress the peak of the compound eluting very close to its formation, while the other peaks eluting earlier from the chromatographic column remain uncompressed. Such a possibility of compressing the isocratic peaks has been already reported in the literature, when the propagation of the speed of mobile phase gradient becomes smaller than the speed of the mobile phase. ${ }^{27-29}$

The start of the front of sample diluent is dependent on its hydrophobicity and the composition of the mobile phase. The retention of sample diluent on the surface of the stationary phase increases with the increase of its hydrophobicity and the decrease of the methanol content in th mobile phase composition. An example of this behaviour is shown in Fig. 12, for the same studied analytes injected in hexanol and using the same mobile phase composition as previously used. The desorption of sample diluent from the stationary phase surface is the opposite situation as the retention. This can explain why octanol does not induce peak compression, as this sample diluent requires high content of methanol in mobile phase composition and elutes from the chromatographic column at longer retention time than the studied analytes.

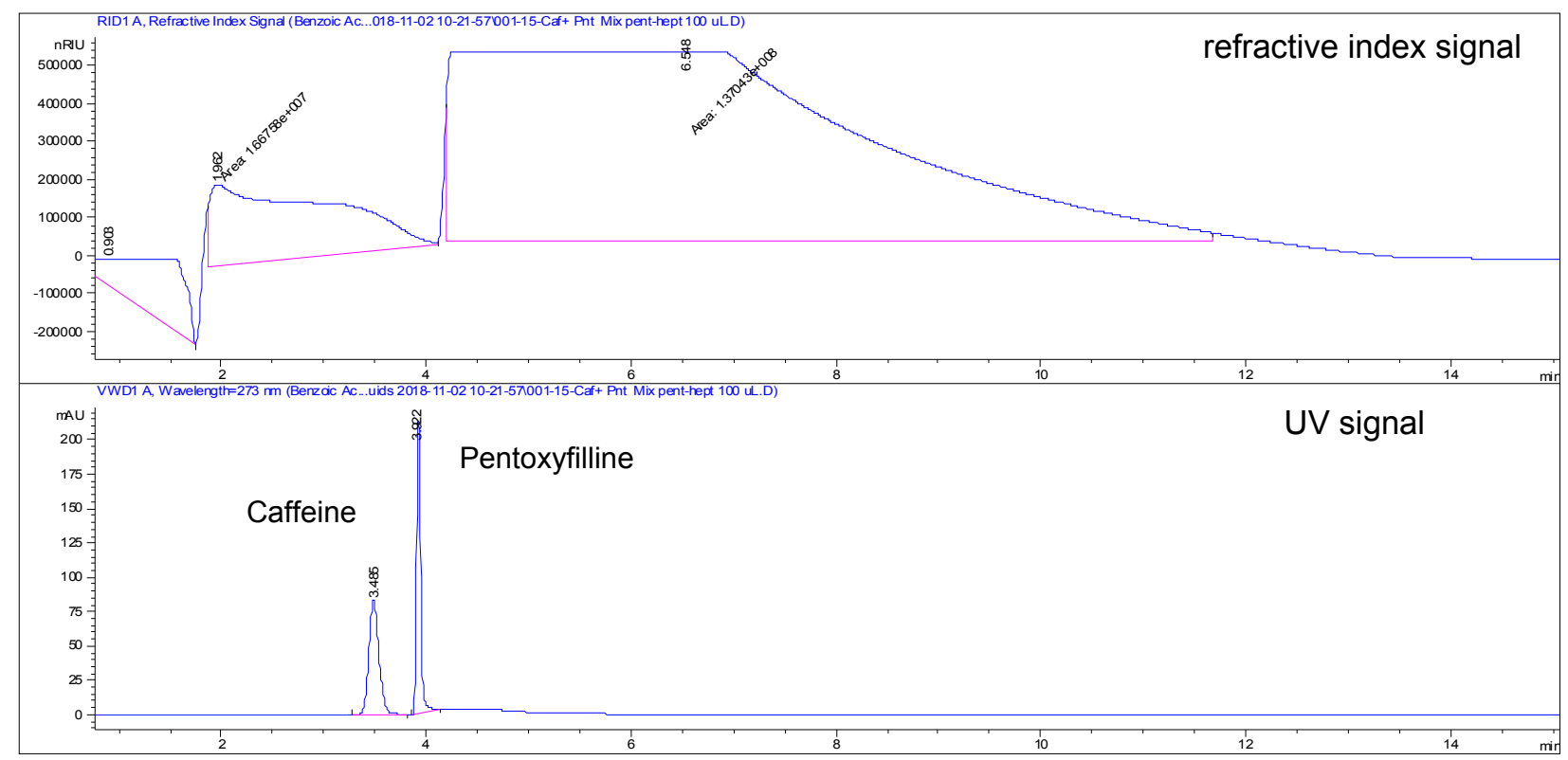

Fig. 11 - Chromatogram of a sample containing caffeine and pentoxyfilline in pentanol, with the injection volume of $100 \mu \mathrm{L}$ (separation performed on a Xterra RP18 column and isocratic conditions water/methanol 65/35, v/v. Upper trace is the refractive index response, and lower trace the UV absorption at $254 \mathrm{~nm}$. 


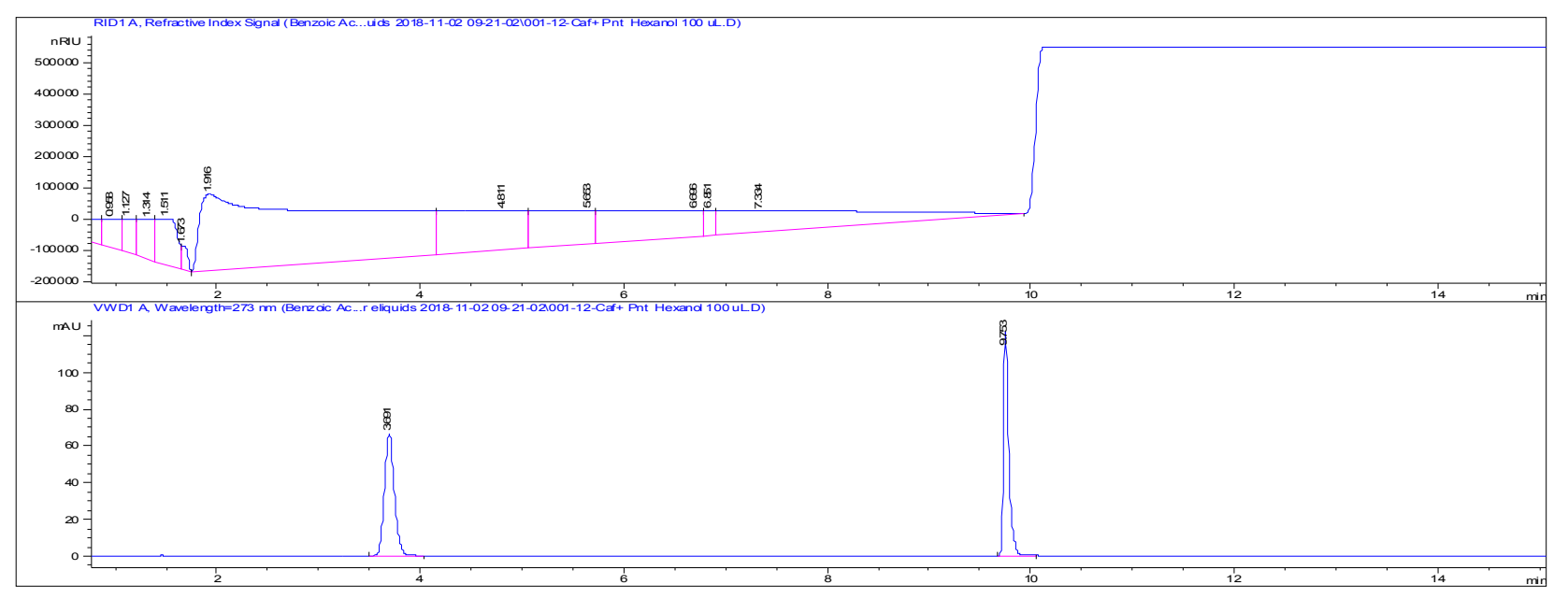

Fig. 12 - Chromatogram of a sample containing caffeine and pentoxyfilline in hexanol, with the injection volume of $100 \mu \mathrm{L}$ (separation performed on a Xterra RP18 column and isocratic conditions water/methanol 65/35, v/v. Upper trace is the refractive index response, and lower trace the UV absorption at $254 \mathrm{~nm}$.

Peak splitting was observed to occur for small injection volumes (up to $50 \mu \mathrm{L}$ ), when the analyte is concentrating in the front of solvent during the adsorption of the sample solvent on the stationary phase surface (Figures 2,3,4,8,9). As mentioned at the Experimental section, the amount of analyte loaded into the chromatographic column was kept constant for all experiments involving different injection volumes. By concentrating in the front of solvent, the analyte molecules enter the adsorption competition with the solvent molecules, and thus the analyte is divided into two fractions: one already adsorbed on the stationary phase and the other still present in the mobile phase. The last fraction of analyte molecules will participate to the partition process between mobile and stationary phase soon after the solvent is entirely adsorbed on the stationary phase surface.

\section{CONCLUSIONS}

Injection of high volumes of samples containing hydrophobic alcohols (butanol to octanol series) induced a decrease of the retention of studied compounds (pharmaceuticals, pollutants and natural), and in some situations a peak compression was observed. Among them, only pentanol, hexanol and heptanol were observed to produce peak compression resulting in a high chromatographic efficiency for the studied compounds, while butanol and octanol allowed large volume injection with gradual decrease of the retention time of the dissolved analytes, but without their peak compression.

Pentoxifylline, salicilic acid, simasine, are some examples of analytes that are characterized by sharp chromatographic peaks (high separation efficiency, over 10,000 plates/column), when high volumes of pentanol or hexanol $(50-100 \mu \mathrm{L})$ solutions are injected. Chromatogram monitoring was performed by UV-spectrometry detection for the studied analytes or refractive index detection, which indicates the front of the sample diluent eluting from the chromatographic column. The front of the sample diluent may influence the compression of the analytes that are eluting very close to its start.

\section{REFERENCES}

1. R. M. Smith, J. Chromatogr. A, 2003, 1000, 3-27.

2. S. C. Moldoveanu, J. Chromatogr. Sci., 2004, 42, 1-14.

3. Y. Chen, Z. Guo, X. Wang and C. Qiu, J. Chromatogr. A, 2008, 1184, 191-219.

4. H. Y. Kaw, J. Li, X. Jin, Z. Wang, L. Cong, M. He and D. Li, Analyst, 2018, 143, 4575-4584.

5. M. E. Padrón, C. Afonso-Olivares, Z. Sosa-Ferrera and J. J. Santana-Rodríguez, Molecules, 2014, 19, 10320 10349.

6. L. J. Fitzpatrick and J. R. Dean, Anal. Chem., 2002, 74, 74-79.

7. H. Kataoka, TrAC - Trends Anal. Chem., 2003, 22, 232244.

8. D. T. Rossi and K. G. Miller, Sep. Sci. Technol., 2004, 5, 165-201.

9. F. R. Mansour and M. A. Khairy, J. Chromatogr. B Anal. Technol. Biomed. Life Sci., 2017, 1061-1062, 382-391.

10. A. Medvedovici, E. Bacalum and V. David, Biomed. Chromatogr., 2018, 32, e4137.

11. Y. Q. Tang and N. Weng, Bioanalysis, 2013, 5, 15831598.

12. J. Zhang, H. Wu, E. Kim and T. A. El-Shourbagy, Biomed. Chromatogr., 2009, 23, 419-425.

13. A. Medvedovici, V. David, V. David and C. Georgita, J. Liq. Chromatogr. Rel. Technol., 2007, 30, 199-213. 
14. S. Udrescu, A. Medvedovici and V. David, J. Sep. Sci., 2008, 31, 2939-2945.

15. S. Udrescu, I. D. Sora, V. David and A. Medvedovici, J. Liq. Chromatogr. Rel. Technol., 2010, 33, 133-149.

16. P. Lazar, S. Udrescu, F. Tache, F. Albu, N. Grinberg and A. Medvedovici, Anal. Methods, 2015, 7, 342-352.

17. T. Galaon, E. Bacalum, M. Cheregi and V. David, J. Chromatogr. Sci., 2013, 51, 166-172.

18. S. Udrescu, I. D. Sora, F. Albu, V. David and A. Medvedovici, J. Pharm. Biomed. Anal., 2011, 54, 1163-1172.

19. V. David, T. Galaon and H. Y. Aboul-Enein, J. Chromatogr. A, 2014, 1323, 115-122.

20. E. Loeser, S. Babiak and P. Drumm, J. Chromatogr. A, 2009, 1216, 3409-3412.
21. A. Medvedovici, S. Udrescu and V. David, Biomed. Chromatogr., 2013, 27, 48-57.

22. U. D. Neue, D. H. Marchand and L. R. Snyder, J. Chromatogr. A, 2006, 1111, 32-39.

23. F. Gritti and G. Guiochon, J. Chromatogr. A, 2008, 1212, 35-40.

24. U. D. Neue, J. Chromatogr. A, 2005, 1079, 153-161.

25. F. Gritti and G. Guiochon, J. Chromatogr. A, 2008, 1215, 64-73.

26. S. C. Moldoveanu and V. David, "Essentials in Modern HPLC Separations", Elsevier, Amsterdam, 2013.

27. F. Gritti, J. Chromatogr. A, 2016, 1433, 114-122.

28. U. D. Neue, D. H. Marchand and L. R. Snyder, J. Chromatogr. A, 2006, 1111, 32-39.

29. W. Hao, K. Wang, B. Yue, Q. Chen, Y. Huang, J. Yu and D. Li, J. Chromatogr. A, 2020, 1619, 460908. 